\title{
MicroRNA profiling of human kidney cancer subtypes
}

\author{
DAVID PETILLO ${ }^{1}$, ERIC J. KORT ${ }^{1,2}$, JOHN ANEMA $^{4}$, KYLE A. FURGE $^{3}$, XIMING J. YANG $^{5}$ and BIN TEAN TEH ${ }^{1,6}$ \\ Laboratories of ${ }^{1}$ Cancer Genetics, ${ }^{2}$ Epidemiology, ${ }^{3}$ Computational Biology, Van Andel Research Institute; ${ }^{4}$ Department of \\ Urology, Spectrum Health Hospital, Grand Rapids, MI 49503; ${ }^{5}$ Department of Pathology, Feinberg School of Medicine \\ Northwestern University Feinberg School of Medicine, Chicago, IL 60611, USA; ${ }^{6}$ NCCS-VARI \\ Translational Cancer Research Laboratory, National Cancer Centre, 169610 Singapore
}

Received March 10, 2009; Accepted April 13, 2009

DOI: 10.3892/ijo_00000318

\begin{abstract}
Although the functions of most of the identified microRNAs (miRNAs) have yet to be determined, their use as potential biomarkers has been considered in several human diseases and cancers. In order to understand their role in renal tumorigenesis, we screened the expression levels of miRNAs in four subtypes of human renal neoplasms: clear cell, papillary, and chromophobe renal cell carcinomas (RCC) as well as benign renal oncocytomas. We found a unique miRNA signature for each subtype of renal tumor. Furthermore, we identified unique patterns of miRNA expression distinguishing clear cell RCC cases with favorable vs. unfavorable outcome. Specifically, we documented the overexpression of miRs 424 and 203 in clear cell RCC relative to papillary RCC, as well as the inversion of expression of miR-203 in the benign oncocytomas (where it is underexpressed relative to normal kidney) as compared to the malignant chromophobe RCC (where it is overexpressed relative to normal kidney). Our results further suggest that overexpression of S-has-miR-32 is associated with poor outcome. While previous studies have identified unique miRNA expression pattern distinguishing tumors from different anatomical locations, here we extend this principle to demonstrate the utility of miRNA expression profiling to identify a signature unique to various tumor subtypes at a single anatomic locus.
\end{abstract}

\section{Introduction}

MicroRNAs (miRNAs) are a class of small non-coding RNAs which have recently received intense interest for their ability

Correspondence to: Dr David Petillo or Dr Bin Tean Teh, Laboratory of Cancer Genetics, Van Andel Research Institute, 333 Bostwick Avenue NE, Grand Rapids, MI 49503, USA

E-mail: bin.teh@vai.org

Abbreviations: miRNA, microRNA; mRNA, messenger RNA; $\mathrm{RCC}$, renal cell carcinoma; $\mathrm{CGH}$, comparative genomic hybridization; PGR, photogenerated reagent

Key words: microRNA, profiling, kidney cancer to regulate gene expression in a variety of cellular processes, including developmental timing (1), cell differentiation (2), cell death (3), cancer development (4), and viral regulation (5). The seed region (bases 2-8, numbered from the 5'-end) of mature miRNAs binds to sequences in the 3 '-untranslated regions of specific messenger RNAs (mRNAs), causing their cleavage or, more commonly, repressing protein translation (6). The expression levels of miRNAs in human cancers have recently been described for several tumors, including renal cell carcinoma $(\mathrm{RCC})(7,8)$. In the case of poorly differentiated tumors, miRNA profiling has been shown to be more robust with regard to classification than mRNA expression profiling (8-10). We extend this work by reporting for the first time miRNA expression profiles of several common renal tumor subtypes.

In order to understand the role of miRNA in renal tumorigenesis, we studied the most common human renal neoplasms which are clear cell RCC (ccRCC), papillary RCC, chromophobe RCC and the closely related benign tumor oncocytoma. In addition, we investigated miRNA expression patterns that distinguish ccRCC cases with poor vs. good prognosis, as this kidney cancer occurs most frequently (75\%) among all subtypes and has the highest rate of local invasion, metastasis, and mortality of the adult renal tumors. These histological subtypes and the prognostic subgroups (i.e., poor and good prognosis) of ccRCC subgroups exhibit distinct histopathological characteristics, clinical behavior, and underlying genetic alterations (11).

\section{Materials and methods}

We selected kidney tumor tissues from 20 patients, four cases from each of the following histological subtypes: oncocytoma, chromophobe, papillary, poor-prognosis clear cell, and goodprognosis clear cell. The prognostic classification of the clear cell samples was based on cancer-specific post-nephrectomy survival of less than 5 years (poor prognosis) or 5 years or more (good prognosis). Total RNA was extracted from these samples and from their corresponding matched normal kidney tissues, with the mirVana ${ }^{\mathrm{TM}}$ miRNA Isolation kit (Ambion, Inc., Austin, TX). All 20 corresponding matched normal tissues were pooled equally, based on total RNA concentration. Samples were obtained from the Spectrum Health Hospital of Grand Rapids, MI and the Cooperative 
Human Tissue Network (CHTN) of the National Cancer Institute. Informed written consent of each patient was obtained, and this study was approved by the Institutional Review Boards of the Van Andel Research Institute and Spectrum Health Hospital.

Microarray. Microarray assay was performed using a service provider (LC Sciences, Houston, TX). The assay started from a $25-\mu \mathrm{g}$ total RNA sample, which was size-fractionated using a YM-100 Microcon centrifugal filter (from Millipore Corp., Billerica, MA), and the small RNAs (less than $300 \mathrm{nt}$ ) isolated were 3 '-extended with a poly(A) tail using poly(A) polymerase. An oligonucleotide tag was then ligated to the poly(A) tail for later fluorescent dye staining; two different tags were used for the two RNA samples in dual-sample experiments. Hybridization was performed overnight on a $\mu$ Paraflo microfluidic chip using a microcirculation pump (Atactic Technologies, Houston, TX) $(12,13)$. On the microfluidic chip, each detection probe consisted of a chemically modified nucleotide coding segment complementary to the target miRNA (from miRBase, http://microrna.sanger.ac.uk/sequences/) and a spacer segment of polyethylene glycol to extend the coding segment away from the substrate. The detection probes were made by in situ synthesis using photogenerated reagent (PGR) chemistry.

The hybridization melting temperatures were balanced by chemical modifications of the detection probes. Hybridization used $100 \mu 1$ of $6 \mathrm{X}$ SSPE buffer $(0.90 \mathrm{mM} \mathrm{NaCl}, 60 \mathrm{mM}$ $\mathrm{Na}_{2} \mathrm{HPO}_{4}, 6$ mM EDTA, pH 6.8) containing $25 \%$ formamide at $34^{\circ} \mathrm{C}$. After hybridization detection, we used fluorescence labeling using tag-specific $\mathrm{Cy} 3$ and $\mathrm{Cy} 5$ dyes. Hybridization images were collected using a laser scanner (GenePix 4000B, Molecular Devices, Sunnyvale, CA) and digitized using ArrayPro image analysis software (Media Cybernetics, Bethesda, MD). Data were analyzed by first subtracting the background and then normalizing the signals using a LOWESS filter (Locally-weighted Regression) (14). For two-color experiments, the ratio of the two sets of detected signals ( $\log 2$ transformed, balanced) and p-values of the t-test were calculated; differentially detected signals were those with p-values of $<0.01$.

Statistical analyses. Prior to clustering, the data were filtered such that miRNAs for which data were missing in more than $50 \%$ of the cases were excluded. Unsupervised average linkage hierarchical clustering was then performed based on the Pearson correlation of the cases.

For discriminant analysis, genes that were differentially expressed between the subtypes were identified using linear modeling as implemented in the Limma package (15) for the $\mathrm{R}$ statistical analysis framework (16). This package is designed to discriminate between samples of only two classes; however, we observed that effective discrimination of all 4 subtypes was achieved when the model was set up to discriminate gene expression of ccRCC vs. all of the other subtypes combined. P-values were adjusted to control the false discovery rate at $5 \%$ (17). For visualization, average linkage hierarchical clustering of the Pearson correlation of the cases was again performed using just these genes. Genes discriminating between favorable and unfavorable ccRCC cases were similarly identified; however, the false discovery rate threshold was increased to $30 \%$ due to the small sample size.

Reverse transcription-PCR analysis. Real-time PCR of miRNAs reverse-transcribed to cDNA was chosen to validate the relative expressions of the following five miRNAs: hsamiR-21, hsa-miR-187, hsa-miR-203, hsa-miR-215, and hsamiR-424. A positive, miR-16, and negative non-human control, ath-miR-156a, were also chosen. Assays were run according to the manufacturer's protocol on a 7700 RT-PCR Sequence Detector (Applied Biosystems, Inc., Foster, CA, USA).

\section{Results}

Unsupervised comparison of multiple kidney cancer subtypes. We first performed an unsupervised hierarchical clustering analysis for all 20 kidney cancer tissues (4 samples per subtype including both good and poor prognosis ccRCC). We selected those miRNAs for which microarray data were measurable in at least $50 \%$ of the samples. The results of this analysis indicate that each class of renal tumors exhibits distinct global miRNA expression patterns (Fig. 1). One exception was a chromophobe sample that clustered among the papillary samples. We reviewed histology reports and found that this chromophobe case had sarcomatoid differentiation, which may account for its atypical miRNA expression signature.

Supervised analysis of multiple kidney cancer subtypes. We next performed discriminant analysis to identify miRNAs that were differentially expressed among the various tumor subtypes. Previous analysis of mRNA expression data has indicated that the greatest genomic similarity exists between chromophobe RCC and oncocytomas, and between papillary RCC and ccRCC. Therefore, we focused on these two comparisons to identify miRNA that could distinguish these pairs of tumor subtypes.

After adjustment of p-values to control false discovery at $5 \%$, five miRNA were identified that significantly differed in expression between chromophobe RCC and oncocytoma samples (Table I and Fig. 2A). The expression levels of the miRNA most overexpressed in chromophobe RCC relative to the oncocytoma tumor samples, miR-203, was confirmed by quantitative RT-PCR (Fig. 2B).

Similarly, 27 miRNAs were identified which exhibited significantly differential expression between ccRCC and papillary RCC samples (Table II and Fig. 3A). Once again, miR-203 exhibited the largest fold change between these two groups of samples (Fig. 2A). We also confirmed the expression levels of the second most overexpressed miRNA in ccRCC relative to papillary RCC, miR-424, by quantitative RT-PCR (Fig. 3B).

Favorable and unfavorable prognostic groups of clear cell carcinoma. We explored miRNAs in clear cell renal carcinoma with respect to their ability to distinguish favorable and unfavorable prognostic groups. Four individual samples were analyzed for each group. Matched normal tissues for all patients in this study (20) were pooled to determine relative expression levels. Only one miRNA, the primary transcript 


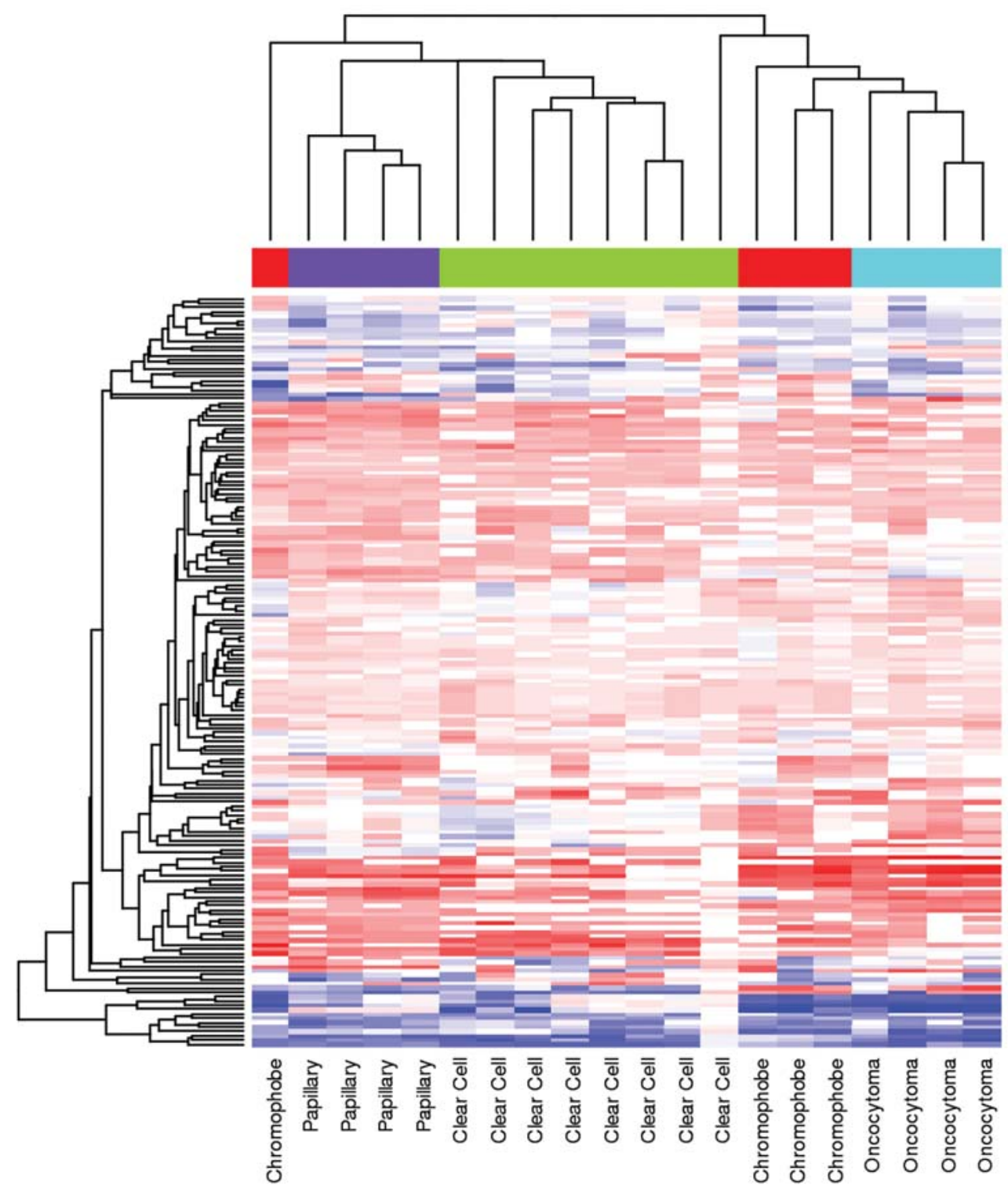

Figure 1. Unsupervised clustering based on miRNA probes present in more than $50 \%$ of cases. Blue areas represent miRNA levels underexpressed relative to the pooled normal renal tissues (derived from matched, individual samples, $\mathrm{n}=20$ ); red areas represent miRNA levels that are overexpressed.

Table I. miRNAs discriminating chromophobe RCC and oncocytomas (false discovery $<5 \%$ ). ${ }^{\text {a }}$

\begin{tabular}{lcc}
\hline microRNA & Chr: Onc ratio $(\log )$ & p-value \\
\hline hsa-miR-203 & 4.49 & 0.00080 \\
S-hsa-mir-200b & 2.89 & 0.00046 \\
S-hsa-mir-197 & 1.99 & 0.00041 \\
hsa-miR-320 & 1.50 & 0.00058 \\
hsa-miR-186 & -2.88 & 0.00078 \\
\hline
\end{tabular}

${ }^{a}$ Average-fold change (chromophobe vs. oncocytoma) and unadjusted p-value for 5 miRNAs.

for miR-32, was identified by this analysis with a predicted false discovery rate less than $5 \%$. However, 20 miRNAs exhibited differential expression with unadjusted $\mathrm{p} \leq 0.05$ (Fig. 4 and Table III). Observed changes in poor prognosis cases relative to good prognosis cases included relative overexpression of S-hsa-miR-32 and S-hsa-miR-342, as well as, relative underexpression of hsa-miR-130a and S-hsamiR-30c-2.

\section{Discussion}

Recently published work has demonstrated that, similar to messenger RNA, tumors from different anatomical sites have unique patterns of microRNA expression. Furthermore, for some poorly differentiated tumors, miRNA expression profiling may be more robust than mRNA expression profiling with respect to classification of these tumors (8) although verification of this finding is necessary.

Renal neoplasms are a group of heterogeneous tumors with distinct histological, biochemical and molecular genetic characteristics, which not only display different prognosis but 
A
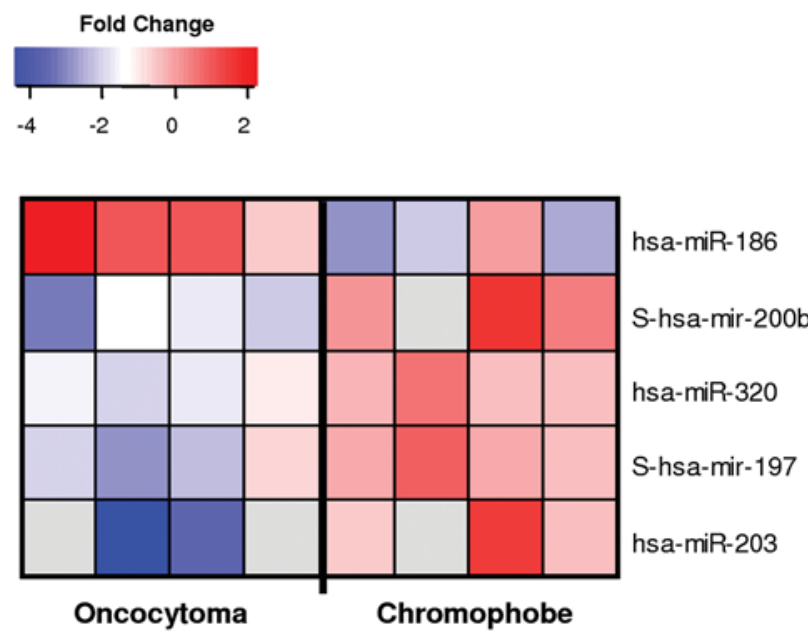

B

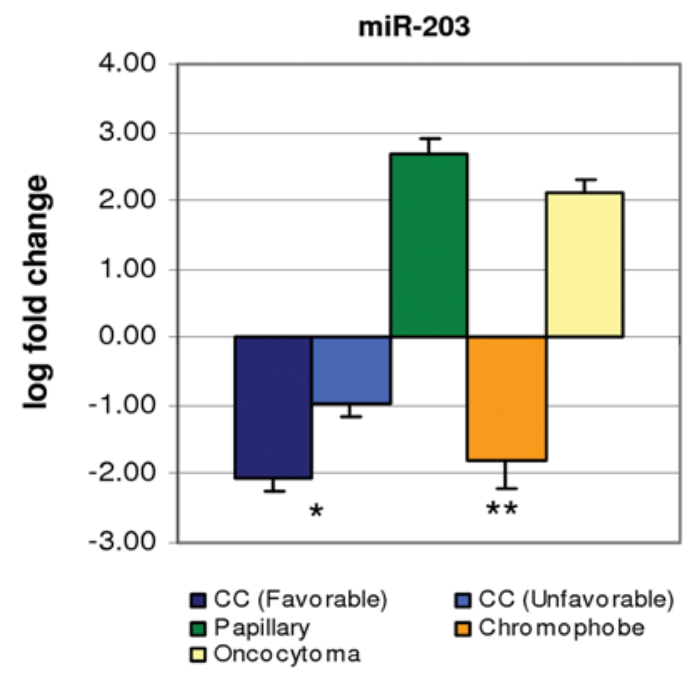

Figure 2. Expression levels of miRNAs discriminating between oncocytoma and chromophobe RCC. (A), Heatmap of expression levels relative to pooled normal kidney. Blue areas represent miRNAs that are underexpressed compared to the pooled normal renal tissues (derived from matched, individual samples, $n=20$ ); red areas represent miRNAs that are overexpressed. (B), Expression levels of miR-203 as measured by quantitative RT-PCR (4 samples analyzed in triplicate for each group). Values represent $\mathrm{Ct}$ values converted to fold change relative to the mean across all groups for visualization. ${ }^{*} \mathrm{p}<0.05$ for ccRCC vs. papillary RCC. ${ }^{* *} \mathrm{p}<0.05$ for chromophobe RCC vs. oncocytoma.

also require different clinical management (18-20). Therefore, it is important to study the miRNA expression patterns of different subtypes of renal cell neoplasms. Gottardo and associates previously examined miRNA expression in renal tumors and bladder tumors and showed that these tumors had distinctive expression profiles as well (7). They hypothesized that this relates to distinct paths to malignancy in these tumors. This is further born out in our study. We observed a number of miRNA expression shifts which may be associated with development of specific tumor subtypes, progression from benign to malignant phenotype, or tumor progression. Examples documented here include the overexpression of miR-424 and miR-203 in ccRCC relative to papillary, as well as the inversion of expression of miR-203 in the benign
Table II. miRNAs discriminating ccRCC and papillary RCC (false discovery $<5 \%$ ).

\begin{tabular}{|c|c|c|}
\hline microRNA & CC: Pap ratio $(\log )$ & p-value \\
\hline hsa-miR-203 & 4.74 & 0.00001 \\
\hline hsa-miR-424 & 4.34 & 0.00081 \\
\hline hsa-miR-450 & 4.16 & 0.00161 \\
\hline hsa-miR-139 & 3.55 & 0.00317 \\
\hline S-hsa-mir-424 & 3.03 & 0.00056 \\
\hline hsa-miR-143 & 3.00 & $<0.00001$ \\
\hline hsa-miR-503 & 2.97 & 0.00255 \\
\hline hsa-miR-224 & 2.94 & 0.00246 \\
\hline hsa-miR-145 & 2.59 & 0.00004 \\
\hline S-hsa-mir-199b & 2.54 & 0.00075 \\
\hline hsa-miR-199ab & 2.48 & 0.00072 \\
\hline hsa-miR-126 & 2.27 & $<0.00001$ \\
\hline S-hsa-mir-224 & 2.17 & 0.00510 \\
\hline hsa-miR-214 & 1.96 & 0.00197 \\
\hline hsa-miR-195 & 1.30 & 0.00067 \\
\hline hsa-miR-324-3p & -0.93 & 0.00478 \\
\hline hsa-miR-191 & -1.13 & 0.00012 \\
\hline hsa-miR-423 & -1.36 & 0.00014 \\
\hline hsa-miR-505 & -1.43 & 0.00471 \\
\hline hsa-miR-425 & -1.50 & 0.00005 \\
\hline S-hsa-mir-149 & -1.55 & 0.00328 \\
\hline S-hsa-mir-328 & -1.68 & 0.00585 \\
\hline S-hsa-mir-365-1 & -2.48 & 0.00388 \\
\hline S-hsa-mir-365-2 & -2.55 & 0.00537 \\
\hline S-hsa-mir-371 & -2.55 & 0.00043 \\
\hline S-hsa-mir-504 & -2.62 & 0.00012 \\
\hline hsa-miR-31 & -4.91 & 0.00140 \\
\hline
\end{tabular}

${ }^{a}$ Average-fold change (ccRCC vs. papillary RCC) and unadjusted p-value for 27 miRNAs. S-prefix indicates primary transcript (premiRNA) vs. mature transcript. ${ }^{b}$ Antisense strand of the mature miRNA sequence.

oncocytoma (where it is underexpressed relative to normal kidney) as compared to the malignant chromophobe RCC (where it is overexpressed relative to normal kidney).

This is the first report we are aware of in the literature of a link between miR-424 and cancer. Previous reports of miR-203 expression in the context of cancer suggest its expression is tissue-type specific. This microRNA has been shown to be underexpressed in esophageal tumors (21), whereas it is overexpressed in both bladder and ovarian tumors $(7,22)$, consistent with our finding that its expression is increased in malignant chromophobe RCC samples, but decreased in the benign oncoytoma samples. An unproven hypothesis exists that oncocytomas can progress into malignant chromophobe RCC. If this proves to be the case, it may be that miR-203 is one of the factors either driving or resulting from this progression by an epigenetic mechanism or by other biological processes. 
A
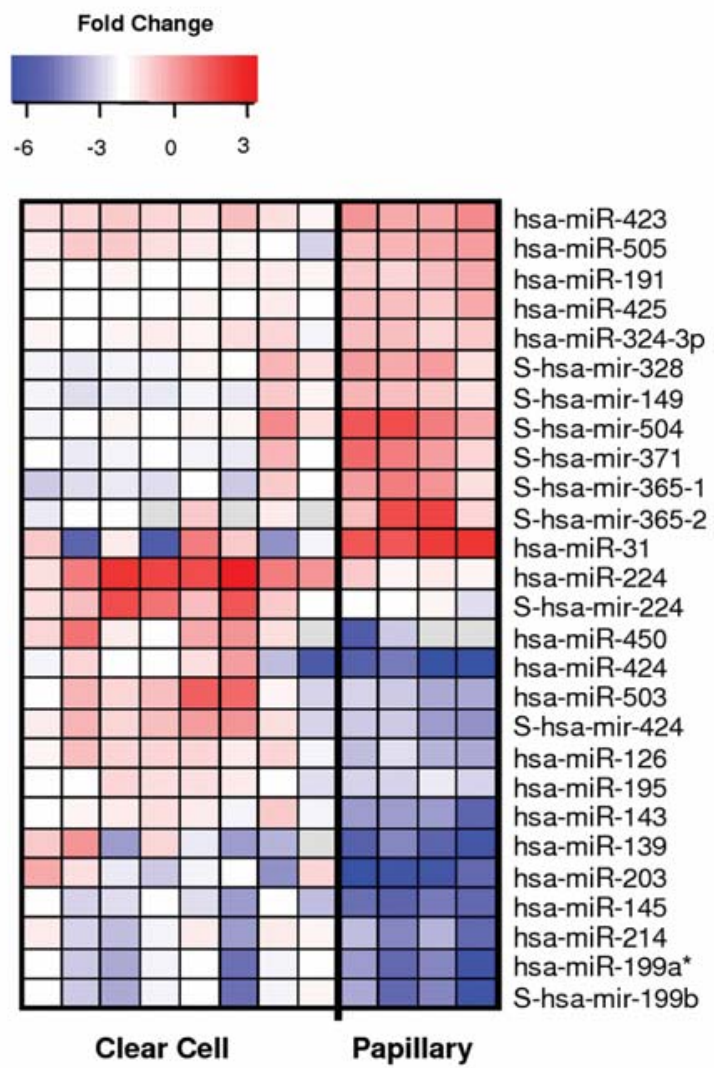

B

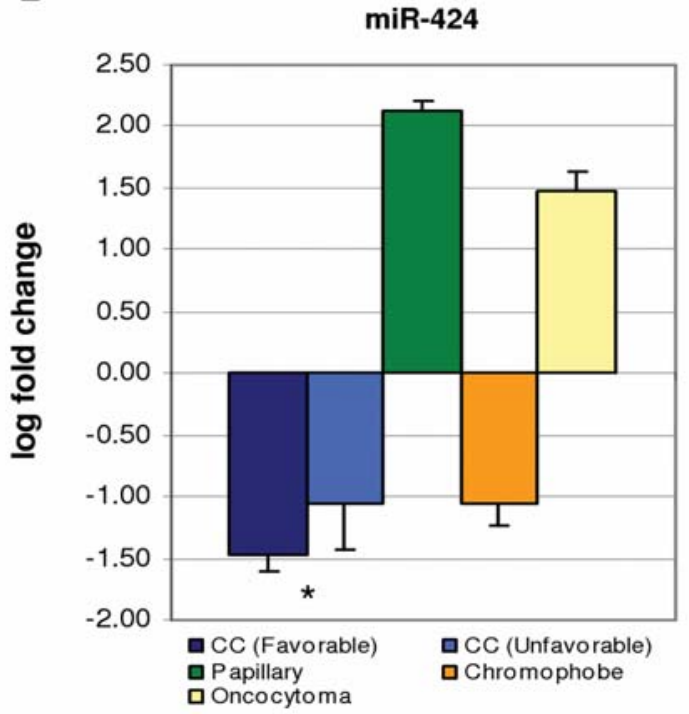

Figure 3. Expression levels of miRNAs discriminating between ccRCC and papillary RCC. (A), Heatmap of expression levels relative to pooled normal kidney. Blue areas represent miRNAs that are underexpressed compared to the pooled normal renal tissues (derived from matched, individual samples, $n=20$ ); red areas represent miRNAs that are overexpressed. (B), Expression levels of miR-424 as measured by quantitative RT-PCR ( 4 samples analyzed in triplicate for each group). Values represent $\mathrm{Ct}$ values converted to fold change relative to the mean across all groups for visualization. ${ }^{*} \mathrm{p}<0.05$ for $\mathrm{ccRCC}$ vs. papillary RCC.

Our results suggest that overexpression of S-hsa-miR-32 (the primary transcript from which miR-32 is formed by the cleaving action of Dicer) is associated with poor outcome.

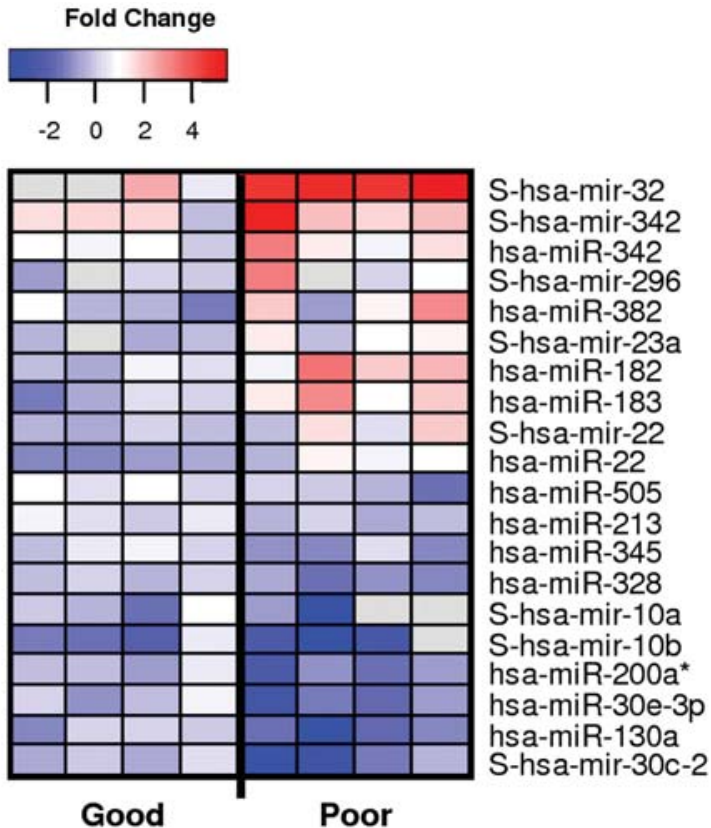

Figure 4. Expression levels of miRNAs discriminating between good and poor prognostic groups of ccRCC. Heatmap of expression levels relative to pooled normal kidney. Blue areas represent miRNAs that are underexpressed compared to the pooled normal renal tissues (derived from matched, individual samples, $\mathrm{n}=20$ ); red areas represent miRNAs that are overexpressed.

Table III. miRNAs discriminating prognostic subgroups of ccRCC (unadjusted $\mathrm{p} \leq 0.05$ ). ${ }^{\mathrm{a}}$

\begin{tabular}{lcc}
\hline microRNA & Poor: Good ratio (log) & p-value \\
\hline S-hsa-mir-32 & 3.43 & 0.00 \\
hsa-miR-22 & 1.53 & 0.00 \\
S-hsa-mir-30c-2 & -1.85 & 0.01 \\
hsa-miR-182 & 2.05 & 0.01 \\
hsa-miR-200ab & -1.20 & 0.01 \\
hsa-miR-328 & -0.96 & 0.02 \\
hsa-miR-130a & -1.71 & 0.02 \\
hsa-miR-30e-3p & -1.51 & 0.02 \\
S-hsa-mir-22 & 1.25 & 0.02 \\
hsa-miR-345 & -1.03 & 0.03 \\
hsa-miR-505 & -1.11 & 0.03 \\
hsa-miR-342 & 1.12 & 0.03 \\
hsa-miR-183 & 2.17 & 0.04 \\
S-hsa-mir-342 & 1.54 & 0.04 \\
S-hsa-mir-10a & -1.96 & 0.04 \\
hsa-miR-213 & -0.64 & 0.05 \\
hsa-miR-382 & 1.68 & 0.05 \\
S-hsa-mir-10b & -1.79 & 0.05 \\
S-hsa-mir-296 & 1.70 & 0.05 \\
S-hsa-mir-23a & 1.22 & 0.05 \\
\hline
\end{tabular}

${ }^{a}$ Average-fold change (poor prognosis vs. good prognosis) and unadjusted p-value for 20 miRNAs. S-prefix indicates primary transcript (pre-miRNA) vs. mature transcript. ${ }^{\mathrm{b}}$ Antisense strand of the mature miRNA sequence. 
While this miRNA has been previously shown to be overexpressed in prostate tumor tissue relative to normal prostate (16), we are aware of no previous report of the possible utility of this microRNA as a prognostic marker.

To summarize, this is the first study to report the differential expression of microRNAs in different subtypes of renal cell neoplasms. The results from our miRNA profiling of various subtypes of renal cancer demonstrates that not only do miRNAs vary in their expression between tumors of different anatomical location, but also among multiple tumor subtypes within a single location. Furthermore, we also found distinct miRNA patterns correlated to good and poor prognosis ccRCC subtypes. Further studies will clarify the functional consequences, both in terms of clinical behavior and tumor biology, of the miRNA expression patterns we have identified in this study.

\section{Acknowledgements}

We thank Xiaochuan Zhou and Chris Hebel of LC Sciences, Inc. for their technical assistance with the microRNA arrays. We thank the Cooperative Human Tissue Network (CHTN) of the National Cancer Institute for providing samples for analysis. The corresponding authors gratefully acknowledge the generosity of the Van Andel Foundation, The Gerber Foundation, the Hauenstein Foundation, and the Michigan Economic Development Corporation and the Michigan Technology Tri-Corridor for their continued support of this project. We also thank Mr. David Nadziejka for manuscript proofreading; and Ms. Sabrina Noyes for preparing and submitting the manuscript.

\section{References}

1. Chen CZ, Li L, Lodish HF and Bartel DP: MicroRNAs modulate hematopoietic lineage differentiation. Science 303: 83-86, 2004.

2. Esau C, Kang X, Peralta E, et al: MicroRNA-143 regulates adipocyte differentiation. J Biol Chem 279: 52361-52365, 2004

3. Xu P, Guo M and Hay BA: MicroRNAs and the regulation of cell death. Trends Genet 20: 617-624, 2004.

4. Esquela-Kerscher A and Slack FJ: Oncomirs - microRNAs with a role in cancer. Nat Rev Cancer 6: 259-269, 2006.
5. Cullen BR: Viruses and microRNAs. Nat Genet 38 (Suppl 1): S25-S30, 2006.

6. Lewis BP, Shih IH, Jones-Rhoades MW, Bartel DP and Burge CB: Prediction of mammalian microRNA targets. Cell 115: 787-798, 2003.

7. Gottardo F, Liu CG, Ferracin M, et al: Micro-RNA profiling in kidney and bladder cancers. Urol Oncol 25: 387-392, 2007.

8. Lu J, Getz G, Miska EA, et al: MicroRNA expression profiles classify human cancers. Nature 435: 834-838, 2005.

9. Mattie MD, Benz CC, Bowers J, et al: Optimized high-throughput microRNA expression profiling provides novel biomarker assessment of clinical prostate and breast cancer biopsies. Mol Cancer 5: 24, 2006

10. Voorhoeve PM, Le Sage C, Schrier M, et al: A genetic screen implicates miRNA-372 and miRNA-373 as oncogenes in testicular germ cell tumors. Cell 124: 1169-1181, 2006.

11. Yin-Goen Q, Dale J, Yang WL, et al: Advances in molecular classification of renal neoplasms. Histol Histopathol 21: 325-339, 2006.

12. Gao X, Gulari E and Zhou X: In situ synthesis of oligonucleotide microarrays. Biopolymers 73: 579-596, 2004.

13. Zhu Q, Hong A, Sheng N, et al: Microfluidic biochip for nucleic acid and protein analysis. In: Methods Molecular Biology, Rampal JB (ed). Humana Press, Totowa, NJ, 2007.

14. Bolstad BM, Irizarry RA, Astrand M and Speed TP: A comparison of normalization methods for high density oligonucleotide array data based on variance and bias. Bioinformatics 19: 185-193, 2003.

15. Smyth GK: Linear models and empirical bayes methods for assessing differential expression in microarray experiments. Stat Appl Genet Mol Biol 3: Article 3, 2004.

16. Ihaka R and Gentleman R: A language for data analysis and graphics. J Comput Graph Stat 5: 299-314, 1996.

17. Benjamini Y, Drai D, Elmer G, Kafkafi N and Golani I: Controlling the false discovery rate in behavior genetics research. Behav Brain Res 125: 279-284, 2001.

18. Eble JN, Sauter G, Epstein JI and Sesterhenn IA (eds): Pathology and genetics of tumours of the urinary system and male genital organs. In: World Health Organization Classification of Tumours. IARC Press, Lyon, 2004.

19. Takahashi M, Sugimura J, Yang XJ, et al: Molecular subclassification of kidney cancer and the discovery of new diagnostic markers. Oncogene 22: 6810-6818, 2003.

20. Furge KA, Kort EJ, Yang XJ, Stadler WM, Kim H and Teh BT: Gene expression profiling in kidney cancer: combining differential expression and chromosomal and pathway analyses. Clin Genitourin Cancer 5: 227-231, 2006.

21. Feber A, Xi L, Luketich JD, et al: MicroRNA expression profiles of esophageal cancer. J Thorac Cardiovasc Surg 135: 255-260, 2008.

22. Iorio MV, Visone R, Di Leva G, et al: MicroRNA signatures in human ovarian cancer. Cancer Res 67: 8699-8707, 2007. 